\title{
Report of the Research and Survey Committee for Investigation of Illuminating Technology on Mesopic Level
}

Reporter: Katsuto Fujii*

\section{Introduction}

In the mesopic level in which a spectral luminous efficacy function varies complicatedly in relation to a brightness, it was difficult to evaluate the brightness as an accurate psychological quantity based on spectral luminous efficacy so that an attention was not directed so much thereto so far. Recently, such a study as an establishment of spectral luminous efficiency function on the basis of psychology and physiology of eye and a new evaluation system thereof was just started but it is not completed yet.

It is fundamentally important to take it into consideration that a design and an evaluation of illumination should correspond to an actual appearance of visual object so that it is not appropriate to apply the conventional standard of photopic vision to the illumination under the mesopic condition as it is. Therefore, it is concluded that it is necessary to provide separately another standard of evaluation system and lighting system for the mesopic level on the basis of a fundumental study.

This research and survey committee had taken a problem of mesopic level for discussion from the standpoint of illumination engineering and technology. In order to solve this problem, the committee carried out research and survey over 1985 and 1986 according to the following study plan and finally issued a report. This research and survey was executed by a support of Tokyo Electric Power Co., Ltd.

(Study Plan)

(1) Survey of regulation and code concerning lighting installations under a mesopic condition

(2) Fact-finding of lighting installations in mesopic level

* The deceased
(3) Survey of spectral luminous efficacy function in mesopic level and establishment of its tentative value

(4) Discussion of practical photometer (illuminance meter and luminance meter) in mesopic level

(5) Model experiment under the lighting condition in mesopic level

(6) Discussion of standardization of lighting system and evaluation method

An outline of the committee report will be described in the following according to the order of chapters. Concerning the detail, the report should be refered to.

\section{Members of the Committee}

Chairman : K. Fujii (Hosei Univ.)

Secretary : K. Ishine (Tokyo E1. Power Co.) M. Takahashi

S. Takahashi

(Hitachi Illum. Co.)

(Toshiba El. Mat. Co.)

Y. Mishima (Electro Tec. Lab.)

K. Yoshikawa (Matsushita El. Co.)

Member : K. Ikeda (Tokyo Science Univ.)

T. Noguchi (Setsunan Univ.)

K. Takada (Nihon Univ.)

$\mathrm{K}$. Ikegawa

(Kansai El. Power Co.)

S. Ishii (Mitsubishi El. Co.)

K. Kawakami (Iwasaki El. Co.)

J. Inagaki (Koito Ind. Co.)

Y. Kawanaka (Nihon Battery Co.)

H. Yazawa

(Tokyo Opt. Mach. Co.)

T. Iizuka (Matsushita El. Co.)

K. Katori (Electro Tec. Lab.) 
M. Moriyama (Matsushita El. Co.)

S. Kanaya (Matsushita El. Co.)

N. Ishizuka

(Illum. Engng. Inst. Jpn.)

(Working Group)

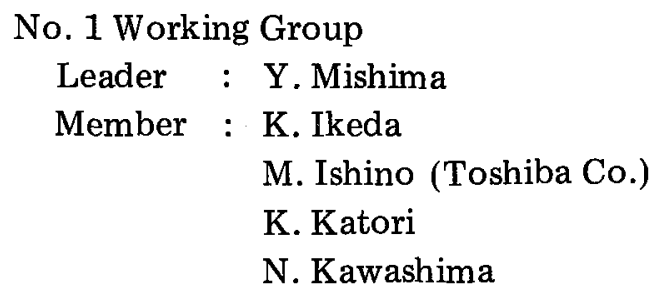

(Kyokko Trading Co.)

T. Kotake (Minolta Camera Co.)

T. Takazawa

(Tokyo Muni. Ind. Tec. Center)

H. Yazawa

S. Horii (Matsushita El. Ind. Co.)

No. 2 Working Group

$\begin{array}{ll}\text { Leader } & \text { S. Takahashi } \\ \text { Subleader: } & \text { J. Inagaki } \\ \text { Member }: & \text { S. Ishii } \\ & \text { K. Kawakami } \\ & \text { T. Jin }\end{array}$

(Jpn. Fire Fighting Res. Inst.)

K. Takada

M. Takahashi

S. Kanaya

Advisor : K. Ikeda

No. 3 Working Group

Leader : K. Yoshikawa

Subleader: Y. Kawanaka

Member : T. Nonaka

K. Ikegawa

T. Iizuka

K. Tanaka

(Hanshin Superhighway Corp.)

K. Toyohiro

(Ohsaka City, Dept. of Park)

A. Shinohara

(Toshiba El. Mat. Co.)

H. Sakai (Iwasaki El. Co.)

K. Watanabe (Seiwa El. Co.)

T. Inagaki (Nihon Battery Co.)

$\mathrm{Y}$. Tanabe

(Matsushita El. Ind. Co.)

3. Survey of Literature and Document concerning Illumination in Mesopic Level

3.1 Survey of Code and Standard concerning Lighting Installations in Mesopic Level
Concerning the lighting installations under the mesopic condition, mainly code and standard of outdoor lighting installations were surveyed and summarized as follows.

\section{(Survey Result)}

Road: For auto road, there are the standards in the Ministry of Construction and the Japan Highway Public Corporation which define the luminance range of $0.5-0.1 \mathrm{~cd} / \mathrm{m}^{2}$.

Street and Passageway: There are standards in JIS, the Ministry of Transport (Civil Aviation Bureau), Japanese Railways and Power Generation and Station Code, almost of which define the illuminance range of $0.2-101 \mathrm{x}$. However, JIS indicates $5-2001 \mathrm{x}$ for street passageway, underground passageway and crossing walkway, in particular, and the Standard of the Ministry of Transport defines $20 \mathrm{~lx}$, especially, for the walkway in the front of the airport terminal.

Square and Parking area: There are standards in the Ministry of Transport, the Japan Highway Public Corporation and JIS, which define the illuminance range of $5-75 \mathrm{~lx}$.

Tunnel and Underground Road: There are standards in the Ministry of Construction and Japanese Railways, which indicate $5 \mathrm{~lx}$ for railway tunnel and $20-60 \mathrm{~lx}$ for road tunnel.

Park: There are the standard in JIS and the design manual in Ohsaka City (Dept. of Park), which indicate $5-30 \mathrm{~lx}$ for main places, $1-$ $10 \mathrm{~lx}$ for other places and $0.3 \mathrm{~lx}$ for children's park.

\subsection{Fact-Finding of Lighting Installations in Mesopic Level}

The survey result of illuminance and light sources for use in the actual roads, squares and parking stations etc. were summarized as follows.

Road: $0.64 \mathrm{~cd} / \mathrm{m}^{2}(7.4 \mathrm{~lx})$ and $1.75 \mathrm{~cd} / \mathrm{m}^{2}$ (42 lx) at two highways; $10 \mathrm{~lx}$ at general road of Kobe Port Island.

Street and Square: $0.5-33 \mathrm{~lx}$ at 10 places.

Parking area: $1-20 \mathrm{~lx}$ at 4 places.

Wharf: $1-50 \mathrm{~lx}$ at 4 wharves.

Walkway: $10-15 \mathrm{~lx}$.

Airport apron: $2-15 \mathrm{~lx}$.

Cave road: $10-20 \mathrm{~lx}$ at 3 places.

Underground road: $20 \mathrm{~lx}$. 
In these lighting installations, HF was mainly used as a light source and was followed by FLR. $\mathrm{NH}, \mathrm{NX}$ and MF etc. are also used.

\subsection{Discussion of Illumination Level of} Lighting Installations corresponding to Mesopic Level

Concerning general lighting, emergency lighting, airport lighting and city lighting, illuminance level was surveyed and it was converted into luminance.

The survey results of $3.1-3.3$ were summarized into tables. Although the illuminance level attained by the outdoor installations is mostly in mesopic level, it is supposed that it takes an important role from the point of safety in society. As a light source, discharge lamp is mostly used because of an importance placed in the power consumption and the spectral distribution of light source, supposedly. It is understood that the illuminance and luminance level of these lighting installations is mostly less than $20 \mathrm{~lx}$ as a mean illuminance and less than 2 $\mathrm{cd} / \mathrm{m}^{2}$ as a mean luminance except a special case. Furthermore, a number of literatures was investigated and they are listed up.

\section{Spectral Luminous Efficiency Function in Mesopic Level}

\subsection{Establishment of Spectral Luminous Ef- ficiency Function in Mesopic Level}

In order to evaluate the brightness in mesopic level as a psychophysical quantity, it is required to establish a spectral luminous efficacy function in mesopic level in the same way as at the evaluation in photopic level. From the abovementioned survey results showing that the luminance level attained by the lighting technology in the mesopic level was $1 \mathrm{~cd} / \mathrm{m}^{2}$ and 0.1 $\mathrm{cd} / \mathrm{m}^{2}$ or there between, it was intended to establish a spectral luminous efficacy function in the adaptation luminance of $1 \mathrm{~cd} / \mathrm{m}^{2}$ and 0.1 $\mathrm{cd} / \mathrm{m}^{2}$. By the way, according to CIE, the mesopic region is shown as an adaptation luminance of $3 \mathrm{~cd} / \mathrm{m}^{2}$ and $0.001 \mathrm{~cd} / \mathrm{m}^{2}$.

\subsection{Determination of Spectral Luminous Efficiency Function in Mesopic Level}

(1) Based on the mean value of the actual measurement data ${ }^{1)}$ in the Electro Technical Laboratory and the Product Science Laboratory, a spectral luminous efficiency function in the mesopic level in the adaptation luminance of 1 $\mathrm{cd} / \mathrm{m}^{2}$ and $0.1 \mathrm{~cd} / \mathrm{m}^{2}$ was determined.

(2) The actual measurement data given in the form of logarithmic scale was converted to a relative luminous efficiency function of mesopic level in linear scale for the convenience of use. It is shown in Fig. 1. The maximum value in the luminous efficacy function, i.e., max. spectral luminous efficacy of $806 \mathrm{~lm} / \mathrm{W}$ (wavelength 540 $\mathrm{nm}$ ) and $892 \mathrm{~lm} / \mathrm{W}$ (wavelength $530 \mathrm{~nm}$ ) was obtained for $1 \mathrm{~cd} / \mathrm{m}^{2}$ and $0.1 \mathrm{~cd} / \mathrm{m}^{2}$, respectively. In the report, the above-mentioned relative luminous efficiency function in the mesopic level at an interval of $5 \mathrm{~nm}$ wavelength and the max. spectral luminous efficacy are given in the form of table.

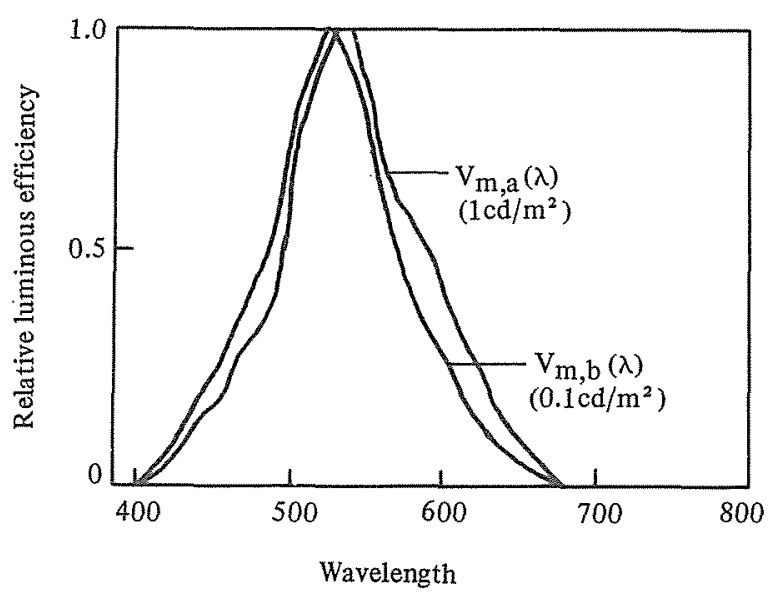

Fig. 1 Relative luminous efficiency function $V_{m, a}(\lambda)$ and $V_{m, b}(\lambda)$ corresponding to adaptation luminance of $1 \mathrm{~cd} / \mathrm{m}^{2}$ and $0.1 \mathrm{~cd} / \mathrm{m}^{2}$

(3) By using a spectral luminous efficiency function for a ten-deg field $K_{10}(\lambda)$ and a spectral luminous efficiency function for tendeg field in the scotopic level (actual measurement) $\mathrm{K}_{\mathrm{b}}(\lambda)$, a spectral luminous efficiency function in the mesopic level was obtained from the following equation. If $\mathrm{K}_{\mathrm{M}}(\lambda)$ means spectral luminous efficiency function in the mesopic level, 
$\log K_{M}(\lambda)=a x \log K_{10}(\lambda)+$

where $b \times \log K_{b}{ }^{\prime}(\lambda)$

$a=0.808 \times L^{0.86} /\left(L^{0.86}+0.027\right)$

$b=0.859 \times 0.0247 /\left(L^{0.86}+0.0247\right)$

$L$ : adaptation luminance in the mesopic level $\left(\mathrm{cd} / \mathrm{m}^{2}\right)$

From this equation, a spectral luminous efficiency function in a desired adaptation luminance can be obtained. The calcurated max. spectral luminous efficacy, i.e., $738 \mathrm{~lm} / \mathrm{W}$ (wavelength $540 \mathrm{~nm}$ ) and $872 \mathrm{~lm} / \mathrm{W}$ (wavelength $530 \mathrm{~nm}$ ) was smaller than the max. spectral luminous efficacy obtained from the actually measured data by $8.4 \%$ and $2.2 \%$ respectively.

(4) Since, in order to evaluate psychologically the brightness in the mesopic level, it is necessary to establish a spectral luminous efficiency function in its level, it was tentatively determined for the convenience of this research and survey. If the spectral luminous efficiency function determined tentatively this time will be employed as a foundation for the determination

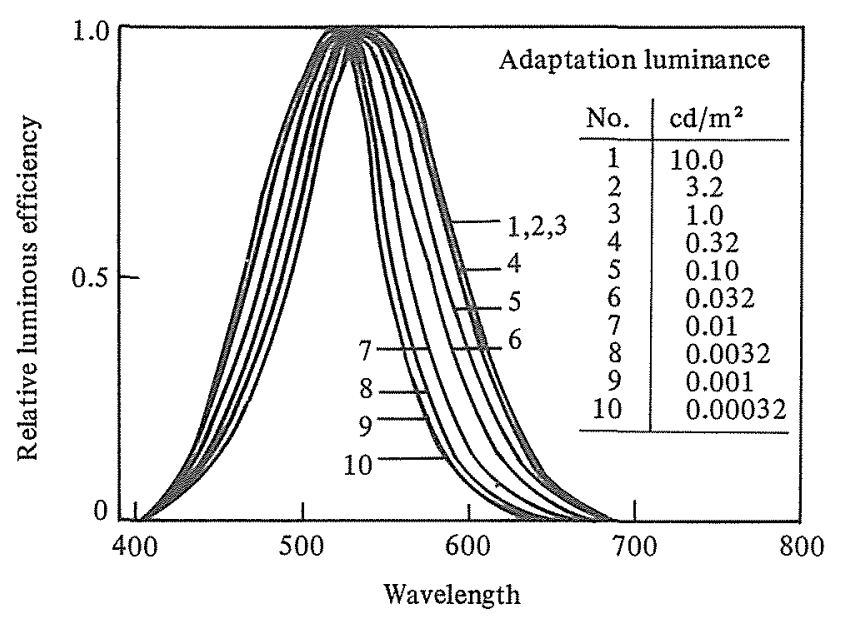

Fig. 2 Result of approximate calcuration by a combination of spectral luminous efficiency for ten-deg. field in photopic level $K_{10}(\lambda)$ and spectral luminous efficiency for ten-deg. field in mesopic level (actual measurement) $K b(\lambda)$

Right side shows relative luminous efficiency of photopic level $\left(10 \mathrm{~cd} / \mathrm{m}^{2}\right)$ and it is rel.lum.eff.function in mesopic level reaching to scopic level $\left(0.001 \mathrm{~cd} / \mathrm{m}^{2}\right)$ by changing the adaptation luminance by an increment of 0.5 of a new spectral luminous efficiency function or for the quantitative evaluation of the brightness in the mesopic level, it is desirable. Fig. 2 shows relative luminous efficiency functions according to an approximate calcuration.

\section{Illuminance Meter and Luminance Meter for Experiment in Mesopic Level}

It was discussed whether it is simple to manufacture the illuminance meter having a relative spectral responsivity approximate to the relative luminous efficiency function in the mesopic level.

(1) Since the luminance meter must take a measurement at a lower luminance level, TOPCON color luminance meter BM-5 using photomultiplier was taken for discussion. By inserting the photopic filter, the mesopic filter (for $1 \mathrm{~cd} /$ $\mathrm{m}^{2}$ ) and the mesopic filter (for $0.1 \mathrm{~cd} / \mathrm{m}^{2}$ ) into a filter turret, one unit of luminance meter can take a measurement of luminance in the photopic level or the mesopic level by turning the filter turret. In the selection of filter and the design of optimum thickness of filter, it was considered that the relative spectral responsivity of the photomultiplier shall be approximate to the spectral luminous efficiency function in the mesopic level (the value given by a calculation formula) as possible by measuring the relative spectral responsivity previously.

(2) Concerning illuminance meter, Toshiba PI-301B phototube illuminance meter was taken for discussion and a head corresponding to the adaptation luminance of $1 \mathrm{~cd} / \mathrm{m}^{2}$ and $0.1 \mathrm{~cd} / \mathrm{m}^{2}$ was manufactured, respectively.

(3) Regarding incadescent lamp, 3 band types of fluorescent lamp, fluorescent high pressure mercury lamp, metal halide lamp, high pressure. sodium lamp, a color correction factor for the standard light A was calcurated, respectively. In the luminance meter, the range of color correction factor for the standard light $A$ was $0.989-$ 1.008 and $0.932-1.043$ for the adaptation luminance of $1 \mathrm{~cd} / \mathrm{m}^{2}$ and $0.1 \mathrm{~cd} / \mathrm{m}^{2}$, respectively. In the illuminance meter, the range of color correction factor the standard illuminants A was $0.965-1.036$ and $0.976-1.033$ for the adaptation luminance of $1 \mathrm{~cd} / \mathrm{m}^{2}$ and $0.1 \mathrm{~cd} / \mathrm{m}^{2}$, respectively. 
(4) It was discovered that it was not so difficult to make the spectral responsivity of the luminance meter and the illuminance meter approximate to the spectral luminous efficiency function in the mesopic level (the value given by a calculation formula).

In the case of luminance meter for the mesopic level, it is rather the problem whether the sensitivity of the photodetector is satisfactory and at the present time it is almost impossible to obtain a satisfactory sensitivity by silicon photodiode.

\section{Evaluation of Brightness of Various} Light Sources in Mesopic Level

\subsection{Comparison of Total Luminous Flux of Various Light Sources in Photopic Level and in Mesopic Level (by Calcuration)}

The total luminous flux was calcurated in accordance with the following formula. By assuming that the law of additivity is applicable to the calcuration formula of total flux in the mesopic level, the formula was established by using an expression similar to the form of the calculation formula in the photopic level.

$$
\begin{aligned}
\text { Photopic : } \phi_{v}=K_{m} \int_{0}^{\infty} \phi_{e, \lambda}(\lambda) V(\lambda) \mathrm{d} \lambda \\
\text { Mesonic : } \phi_{\nu, a}=K_{m, a} \int_{0}^{\infty} \phi_{e, \lambda}(\lambda) V_{m, a}(\lambda) \mathrm{d} \lambda \\
\phi_{\nu, a}=K_{m, a} \int_{0}^{\infty} \phi_{e, \lambda}(\lambda) V_{m, b}(\lambda) \mathrm{d} \lambda
\end{aligned}
$$

where $\phi_{\nu}$ : Total flux of light source in photopic level

$\phi_{v, a}$ : Total flux of light source in adaptation luminance of $1 \mathrm{~cd} / \mathrm{m}^{2}$

$\phi_{\nu, b}$ : Total flux of light source in adaptation luminance of $0.1 \mathrm{~cd} /$ $\mathrm{m}^{2}$

$K_{m}$ : Max. spectral luminous efficacy in photopic level $(683 \mathrm{~lm} / \mathrm{W})$

$K_{m, a}$ : Max. spectral luminous efficacy in adaptation luminance of $1 \mathrm{~cd} /$ $\mathrm{m}^{2}(806 \mathrm{~lm} / \mathrm{W})$

$K_{m, b}$ : Max. spectral luminous efficacy in adaptation luminance of 0.1 $\mathrm{cd} / \mathrm{m}^{2}(892 \mathrm{~lm} / \mathrm{W})$

$\phi_{e, \lambda}$ : Spectral radiant flux of light source

$V(\lambda)$ : Spectral luminous efficiency

Table 1 Comparison of total luminous flux of light source for each spectral luminous efficiency

\begin{tabular}{l|r|r|r|r|c}
\hline \multirow{2}{*}{ Type of lamp } & \multicolumn{5}{|c}{ Relative luminous efficiency } \\
\cline { 2 - 6 } & $V(\lambda)$ & $M a(\lambda)$ & $M b(\lambda)$ & $\begin{array}{c}V m, a(\lambda) \\
\left(1 \mathrm{~cd} / \mathrm{m}^{2}\right)\end{array}$ & $\begin{array}{c}V m, b(\lambda) \\
\left(0.1 \mathrm{~cd} / \mathrm{m}^{2}\right)\end{array}$ \\
\hline Standard & 100.0 & 100.4 & 99.7 & 105.1 & 101.0 \\
illuminants A & 100.0 & 100.4 & 99.8 & 105.1 & 101.0 \\
LW100V95W & 100.0 & 102.7 & 104.0 & 104.1 & 103.1 \\
FL20SS·W/18 & 100.0 & 108.1 & 114.1 & 111.8 & 114.2 \\
FL20S.W-DL-X & 100.0 & 108.8 & 116.1 & 115.2 & 117.6 \\
FL20SS·EX/18 & 100.0 & 96.6 & 92.9 & 102.3 & 95.4 \\
BFG17EX-L & 100.0 & 107.5 & 113.5 & 113.3 & 114.9 \\
BFG17EX-N & 100.0 & 99.1 & 97.6 & 101.3 & 97.1 \\
HF100X & 100.0 & 102.6 & 103.2 & 104.9 & 102.4 \\
MF100-L & 100.0 & 85.6 & 90.0 & 86.1 & 70.5 \\
NH75F.L &
\end{tabular}

(1) $M a(\lambda) \& M b(\lambda):$ Rel.lum.effi, according to the approx. formula $V_{m, a}(\lambda) \& V_{m, b}(\lambda)$ : Rel.lumi.effi. according to the mean of actual measurement

(2) The calcuration was performed by using each rel.lum.effi. and max. spec. lum. effi. (Spec.lum.effi. $=$ Rel.lum.effi. $\times$ Max.spec.lum.effi.)

(3) All of light sources were normalized by $100=$ total luminous flux in photopic level. 


\section{function in photopic level \\ $V_{m, b}:$ Spectral luminous efficiency \\ function in adaptation luminance of $1 \mathrm{~cd} / \mathrm{m}^{2}$ \\ $V_{m, a} \quad$ Spectral luminous efficiency function in adaptation luminance of $0.1 \mathrm{~cd} / \mathrm{m}^{2}$}

The calculated results are shown in Table 1. In Table 1 , the results obtained by using an approximate formula of spectral luminous efficiency function are shown in parallel additionally.

\subsection{Experiment on the Evaluation of Bright- ness of Light Source by Visual Percep- tion at the Transition from the Photopic Range to the Mesopic Range}

On the photometric bench, in reference to an incandescent lamp with the distribution temperature of $2800 \mathrm{~K}$, a photometric balance was taken between this lamp and several types of discharge lamps in the brightness level of photopic range and mesopic range. In order to reduce the light from the state of photopic brightness to the state of mesopic brightness, light reducing filters (filters) having wide and flat spectral transmittance were selected) were weared on the eyes. Observers were 4 men. The experiment was carried out as follows.

While an observer is steadily looking at the Vshaped white board (an incandescent lamp was placed in the left side of the board and a discharge lamp was placed on the right side of the board), the discharge lamp was moved in order to obtain an equal brightness on the left and right surface of the V-shape and the distance between the discharge lamp and the board was measured at the moment of equal brightness. The illuminance on the white board was calculated by a reciprocal of the square of the measured distance. Fig. 3 shows the summarized observed data of 4 observers representing how the illuminance value changes for the individual lamp between in the photopic range and in the mesopic range. It was assumed that the illuminance is proportional to the total flux of light source.

\subsection{Comparison of Calculation with Experi- ment}

As shown in Fig. 3, it was confirmed that the calculation and the experiment have a trend of matching there between qualitatively. The finding that the variation of the visual experimental result is smaller than the variation of the calculated result could not be made clear. A future study is expectable on this concern.

It is obvious that at the transition from the photopic range to the mesopic range the calculated total flux is increased in the light source having a dominant region of emission spectrum between green and blue and decreased in the light source having a dominant region of emission spectrum in red. It is a key factor for

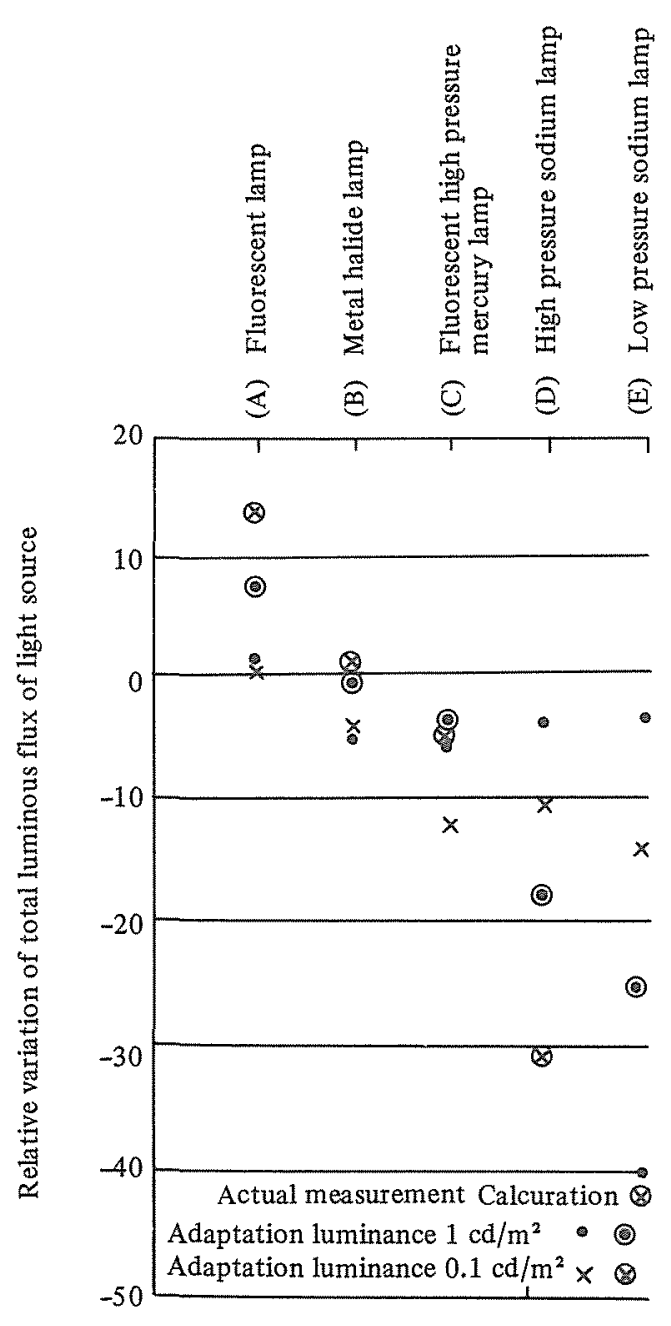

Fig. 3 Relative variation of total luminous flux of light source at the transfer from photopic region to mesopic region (It is shown by relation to the base of incandescent lamp in photopic region and in mesopic region.) 
the increase or the decrease of the total flux whether the light source has a large part of emission spectrum in the wavelength region between 450 and $580 \mathrm{~nm}$.

\section{Model Experiment under the Condition of Lighting in Mesopic Level}

The luminance of chromatic objects depends on the color property, illuminance as well as the spectral distribution of light source for illumination. In order to understand the basic relation between these factors and the visual appearance, several experiments were executed.

\subsection{No. 1 Experiment: Appearance of Color Chip under Fluorescent Lamp}

(1) Purpose

It is the purpose of this experiment to survey the perceived lightness of the color chips illuminated by the fluorescent lamp and to study its correspondence to the value obtained from the spectral luminous efficiency function in the mesopic level.

(2) Date, Location and Others

On Dec. 21 and 22, 1985;

At the Illumination and Quantum Laboratory of Tokyo Science University;

The experiment was performed by No. 1 Working Group with a cooperation of the persons concerned.

(3) Condition and Method 3),4)

The surface of the observation desk and the wall of the laboratory was covered by a gray sheet of N7 entirely and the desk was illuminated by $\mathrm{a}$-highly color rendering fluorescent lamp. The luminance level was set 54 $\mathrm{cd} / \mathrm{m}^{2}$ in the photopic level and $1 \mathrm{~cd} / \mathrm{m}^{2}$ and $0.1 \mathrm{~cd} / \mathrm{m}^{2}$, respectively, in the mesonic level at the measurement in the grey background (N7).

In the measurement, luminance meter, spectrophotometer and spectral luminance meter were used. The luminous reflectance was obtained by multiplying the spectral luminous efficacy function and it was converted into the lightness. As the spectral luminous efficiency function, actually measured spectral luminous efficiency in the photopic region of $V(\lambda)$, in the mesopic region of $1 \mathrm{~cd} / \mathrm{m}^{2}$ and $0.1 \mathrm{~cd} / \mathrm{m}^{2}$ and in the composite spectral luminous efficacy were used. While the observer compares the lightness of the color chip for the observations (45 types of colors with the lightness of 6 and 46 types of color with the lightness of 4) with the lightness of the standard color chip (grey scale) on the desk illuminated by the highly color rendering fluorescent lamp, the observer recorded the scale values of both color chips when he judged the equal lightness.

(4) Result and Discussion

From the values recorded after the judgement of the equal lightness, the following findings were discovered.

(a) In the case of photopic level $\left(54 \mathrm{~cd} / \mathrm{m}^{2}\right)$

Except yellow, the evaluation of lightness is almost constant irrespective of hue. The higher the saturation, the lightness is evaluated more lightly. That is because the color channel might contribute to the sensitivity of the brightness in the photopic level.

(b) In the case between the photopic level and the mesopic level $\left(1 \mathrm{~cd} / \mathrm{m}^{2}\right)$

It is understood that the evaluation of lightness changes little depending on the difference of saturation so that the color channel contributes little to the sensitivity of brightness. Furthermore, in this case, the evaluated value of the lightness depends little on the hue so that it is noted that the sensitivity of brightness by the operation of the cone largely exceeds the contribution by the operation of the rod.

(c) In the case of the mesopic level $(0.1 \mathrm{~cd} /$ $\mathrm{m}^{2}$ )

Due to the increase of the contribution by the operation of the rod, the judgement of lightness changes greatly depending on the hue, that is to say, red and orange appears darker and bluegreen and blue appears brighter. The higher the hue, this trend is more remarkable. This is caused by Purkinje effect. It was made clear quantitative by this experiment.

When the above-mentioned experimental values are compared to the colorimetric value (lightness) calcurated by using the spectral luminous efficacy in the photopic region and the mesopic level, it is understood that in the photopic region a good correlation, with the prior spectral luminous efficacy in the photopic region is noticed and in the mesopic level a good correspondence between the visual brightness and the spectral sensitivity is noticed. 


\subsection{No. 2 Experiment: Appearance of Color Chip under Various Light Source}

(1) Purpose

It is the purpose of this experiment to survey the perceived lightness of the color chip illuminated by a variety of light sources and to study the comparison between the lightness and the physical photometric quantity in the photopic region and in the mesopic region.

(2) Date, Location and Others

On Sept. 19 and 20, 1986;

At Tokyo EC Lighting Laboratory of Matsushita Electric Co.;

This experiment was performed by No. 2 Working Group with a cooperation of the persons concerned.

(3) Condition and Method 3),4)

(a) Observation booth

In the laboratory, there were provided two observation boothes, in which an observation desk was centrally located. The surface of the inner wall and the desk in the booth was covered by grey cloth of $\mathrm{N} 7$.

(b) Illumination level

The luminance level was set $50 \mathrm{~cd} / \mathrm{m}^{2}$ in the photopic level and $1 \mathrm{~cd} / \mathrm{m}^{2}$ and $0.1 \mathrm{~cd} / \mathrm{m}^{2}$, respectively, in the mesopic level at the measurement in the grey background. As the light source, incandescent lamp, fluorescent lamp, fluorescent mercury lamp, metal halide lamp and high pressure sodium lamp were used.

(c) Measuring method

Luminance meter, spectrophotometer and illuminance meter were used. In measuring by the luminance meter, a filter matched to the spectral luminous efficiency of each lighting level was mounted.

(d) Observation method

While the observer compares the lightness of the color chip for the observation with the lightness of the standard color chip (grey scale) in the predetermined lighting level by a variety of light source, the observer recorded the scale values when he judged the equal lightness.

There were prepared totally 47 types of the color chip for the observation with 5 groups of hue $(5 \mathrm{R}, 5 \mathrm{Y}, 5 \mathrm{G}, 5 \mathrm{~B}, 5 \mathrm{P})$ and totally 47 types of the standard color chip (grey scale) with 18 steps from N1 to N9.5 at an interval of 0.5.

(4) Result and Discussion (a) Evaluation of hue and brightness

For the color chip of $5 \mathrm{R}$, in the photopic level the brightness corresponding to its lightness is perceived but in the mesopic level the color chip with lower lightness is perceived relatively darker. On the contrary, for the color chip of $5 \mathrm{~B}$, the chip is appeared relatively brighter in the mesopic level than in the phtopic level.

For the color chip of $5 \mathrm{Y}, 5 \mathrm{G}$ and $5 \mathrm{P}$, a remarkable change was not noticable but it can be seen that $5 \mathrm{Y}$ and $5 \mathrm{P}$ has a tendency slightly resemble to $5 \mathrm{R}$, and $5 \mathrm{G}$ has a tendency slightly resemble to $5 \mathrm{~B}$ (Purkinje effet). According to No.1 Experiment, the higher the hue, Purkinje effect appears more remarkable.

(b) Evaluation of lightness and brightness of color chip

It is assumed that the lightness of the background is 7 . For the color chip of $5 R$, when the lightness is more than 6 or equal, the perception of brightness is almost the same in the mesopic level as in the photopic level under any light source but when the lightness is less than 5 or equal, the perception of brightness in the mesopic level is lowered. The lower the lightness, its tendency is more remarkable (lowering of max. spectral luminous efficacy).

For the color chip of 5B, even in the lightness less than 5 or equal, the brightness is perceived brighter (increase of max. spectral luminous efficacy).

For the color chip of $5 \mathrm{Y}$ and $5 \mathrm{P}$, the tendency resembles to the case of $5 \mathrm{R}$, and in the color chip of $5 \mathrm{~B}$, the tendency resembles to the case of $5 \mathrm{~B}$, although not so remarkable.

From this result and No.1 Experiment, it can be understood that in the mesopic level, if there exist in the present object a color of red-yellow system, its visibility will be lowered.

(c) Type of light source and brightness evaluation

(i) Under the incandescent lamp and three band type fluorescent lamp, the perception of the brightness presents a representative appearance of color in the mesopic level such that the color chip of $5 \mathrm{R}$ and $5 \mathrm{Y}$ with a lower lightness is appeared darker in the mesopic level and, on the contrary, the color chip of $5 \mathrm{~B}$ and $5 \mathrm{G}$ with a higher lightness is appeared brighter in the mesopic level. 
(ii) Under the metal halide lamp, the perception of the brightness is such that there is no difference for blue system such as $5 \mathrm{G}$ and $5 \mathrm{~B}$ between in the mesopic level and in the photopic level. Therefore, it can be said that it is an illumination light source in which the color of red-yellow system does not depend so much on the adaption luminance.

(iii) Under the fluorescent mercury lamp, the perception of the brightness for the color chip of $5 \mathrm{R}$ is lowered generally than under the metal halide lamp. Especially, in the mesopic level the color chip of $5 \mathrm{~B}$ is appeared darker than the color chip of $5 \mathrm{G}$ regardless of the lightness.

The visibility of the color chip of $5 \mathrm{R}$ and $5 \mathrm{P}$ has the same tendency as that of the perception of the brightness under other light sources.

(iv) Under the high pressure sodium lamp, the color chips of $5 \mathrm{Y}$ and $5 \mathrm{~B}$ are perceived always bright and the colors of other hue (especially, 5G and 5B) are perceived always dark.

(d) Judged value and photometric value of lightness

There is a high correlation between the value of lightness of the color chip converted from the luminance measured by the luminance meter having the properly approximated to the spectral luminous efficiency function in the mesopic level and the value of lightness judged by the observer in the mesopic level.

\section{Appearance of Road Sign in the Outdoor Lighting Environment of Mesopic Level}

As an actual example of the mesopic condition, a road-traffic at night was selected and an experiment was executed on the appearance of the road sign in the outdoor, thereby a relation between the light source and the evaluation of the brightness was discussed.

\subsection{Measurement of Visual Acuity under various Light Sources \\ (1) Purpose}

It is the purpose of this experiment to obtain the visual acuity in the photopic level and in the mesopic level in the outdoor.

(2) Date, Location and Others

On Oct. 21, 1986: at 17:00-21:30;

At the Nishinomaru Park of the Ohsaka Castle;
This experiment was performed by No. 3 Working Group with a cooperation of the persons concerned.

(3) Condition and Method

By means of the standard visual acuity table placed in the outdoor, their visual acuity of the observers were measured. The adaption level of the observers was set $50 \mathrm{~lx}, 10 \mathrm{~lx}\left(1 \mathrm{~cd} / \mathrm{m}^{2}\right.$ equivalent) and $1 \mathrm{~lx}\left(0.1 \mathrm{~cd} / \mathrm{m}^{2}\right.$ equivalent $)$ as the vertical illuminance at the location of the visual object. The instruments used in the former experiments was again used. As the light source, incandescent lamp, three band type fluorescent day-white lamp, high pressure sodium lamp and metal halide lamp were used.

(4) Result and Discussion

The difference of the visual acuity depending on the type of light source and the lighting level was not found almost. That is, supposedly, because the visual acuity table is achromatic and the feature of the light source could not be shown due to the reflection light from the cloud.

\subsection{Visibility of Road Sign under Various Light Sources}

(1) Purpose

It is the purpose of this experiment to obtain the difference of the perceptual brightness of the road sign depending on the light source in the outdoor lighting environment.

(2) Date, Location and Others

The same as 8.1.

(3) Condition and Method

The speed limit sign (displaying $40 \mathrm{~km}$ ) placed in the outdoor and its surrounding were lighted by a variety of light sources. The lighting level and the instruments etc. are the same as 8.2.

When the observer judged that the brightness is equal by comparing the road sign lighted by the incandescent lamp and the road sign lighted by other light sources, he expresses the fact of judgement. At that moment the vertical illuminance of both road signs was measured.

(4) Result and Discussion

At the illuminance of $10 \mathrm{~lx}\left(1.0 \mathrm{~cd} / \mathrm{m}^{2}\right.$ equivalent), in order to be visible in the same brightness, a higher illuminance is required in any light source than in the incandescent lamp, especially the high pressure sodium lamp having 
its higher tendency. At the illuminance of $1 \mathrm{~lx}$ $\left(0.1 \mathrm{~cd} / \mathrm{m}^{2}\right.$ equivalent), only the high pressure sodium lamp requires a higher illuminance than the incandescent lamp and the fluorescent lamp has approximately the same brightness as the incandescent lamp. It results that a lower illuminance is sufficient in the metal halide lamp than the incandescent lamp.

The above is a result under the condition in which there is little variation due to the type of light source. Furthermore, it should be noticed that a large difference of visibility among observers was found. It will require a further discussion.

\section{Future Tasks}

\subsection{New Installation of Photometric Sýstem for Mesopic Level}

The photometric quantity in the mesopic level should be evaluated on the basis of its spectral luminous efficacy function. For that purpose, it is necessary to determine the spectral luminous efficacy function in the mesopic level, at first. Considering a practical application, in this research and survey, 2 stages of spectral luminous efficacy functions in the mesopic level were established tentatively. However, it is necessary to complete it by a further study.

Then, based on the determined spectral luminous efficacy function in the mesopic level, an evaluation system of a variety of photometric quantities shall be defined and the symbol representing the photometric quantity and unit shall be defined.

In order to make it obvious that the photometric quantities in the mesopic level is different from the general photometric quantities and uses a different standard, it is necessary to define the expression of symbol and unit separately.

\subsection{Putting Photometric Instrument for Mesopic Level into Practical Use}

In order to survey and evaluate the illumination in the mesopic level, a photometric instrument for the mesopic level is required.

When thinking of putting into practical use, it will be advantageous to use a system in which a color filter is mounted to the photometric instrument for the photopic level, where the color filter is designed and manufactured so as to obtain a spectral responsivity characteristics corresponding to the spectral luminous efficiency function in the mesopic level.

\subsection{On the Appearance of Color}

The general illumination design calculation is directed only to the brightness. From the point of visibility of the object, not only brightness but also visibility of color should be considered as an important factor. As well-known, an evaluation method of the color rendering property of the light source is defined and a color rendering index is officialized for each type of light source, but it has been used only for the secondary purpose in the design aspect.

In this report, the evaluation of the brightness, which is a fundamental element, was studied mainly, but the appearance of color become an important element sometimes as in the case of road sign. Therefore, it is necessary to make a study generally including the appearance of color, hereafter.

\subsection{Discussion on the Standardization of II- lumination Technology in Mesopic Level}

There is a variety of lighting installations apparently corresponding to the mesopic level. They have their own environmental condition and each different illumination purpose so that it is not appropriate to define the lighting system and the evaluation method by a single standard. In order to prepare a preferable illumination, it is necessary to select several representative installations from the indoor and outdoor lighting facilities, respectively, and to execute a practical study individually. At the starting of the study, it is most important to make the environmental condition and the purpose of the illumination obvious and then to obtain a fundamental element and condition required for the appearance. For example, the luminance surface within the visual field relating to the adaptation luminance, the location and the size of the visual object and the surface condition of color and reflectance should be defined concretely. Succeedingly, a lighting system for obtaining a required appearance on the basis of the predetermined conditions shall be studied. In this study, the selection of the type of light source, lighting system and design calculation system etc. are included. In these 
operations, the results obtained by this research and survey will be helpful sufficiently.

Also, in order to perform survey and discussion of the lighting installations, it is required to establish an evaluation method. Relating thereto, the illuminance meter and luminance meter for the mesopic level will take an important role. For that purpose, an application method of the photometric instruments already used practically should be studied.

It is expected that in reference to the abovementioned proposals a preparation of the standard will be realized.

\section{References}

(1) Sagawa, K. and Takeichi, K.: Special luminous efficiency function for a ten-degree field in the mesopic range., J: Light \& Vis. Env. 7, No. 1, 36 (1983)

(2) Fuwa, M. and Katori, K.: Non-linearity of brightness sensitivity and its scale,, Inst. Electri. Engers. Japan, Committee Rept. LAV 82-40 (1982)

(3) Ashizawa, M. and Ikeda, M.: Change of brightness of cloths depending on the illuminance level., J. Illum. Engng. Inst. Jpn. Vol. 67 (1983) 503

(4) Ashizawa, M., Ikeda, M. and Nakano, Y.: Experiment and analysis of change of brightness of color cloths depending on the illuminance level, J. Mlum. Engng. Inst. Jpn. Vol. 69 (1985) 272

Received May 22, 1990; accepted June 26, 1990. 